\title{
The Association between Dietary Total Antioxidant Capacity and Risk of Non Alcoholic Fatty Liver Disease (NAFLD) in Adults: A Case-Control Study
}

Mohammadhassan Sohouli ( $\sim$ mohammadhassansohouli@gmail.com )

Iran University of Medical Sciences

Somaye Fatahi

Iran University of Medical Sciences

Farzad Shidfar

Iran University of Medical Sciences

\section{Research}

Keywords: dietary total antioxidant capacity, Obesity, Chronic Diseases, Non-alcoholic Fatty Liver Disease

Posted Date: April 29th, 2020

DOI: https://doi.org/10.21203/rs.3.rs-24074/v1

License: (c) (i) This work is licensed under a Creative Commons Attribution 4.0 International License.

Read Full License 


\section{Abstract}

\section{Objective}

Dietary total antioxidant capacity (TAC) has been assumed as a useful tool to assess the relationship between the cumulative antioxidant food capacity and several chronic disorders. However, the relationship between the total antioxidant capacity of the diet (TAC) and the risk of NAFLD has not been previously examined. The aim of this study was to evaluate the association between DTAC and risk of NAFLD in a case-control study.

\section{Methods}

This case-control study was carried out among 158 patients with NAFLD and 357 healthy subjects aged 18-55 years. Dietary data were collected using a validated 168- items quantitative food frequency questionnaire. DTAC was calculated based on the ferric reducing-antioxidant power (FRAP) values that reported by US Department of Agriculture.

\section{Results}

The mean age and BMI of the study participants were 43.9 years and 30.5. The NAFLD patients had higher BMI and female's percentage compared with controls. Also, compared with the control, the NAFLD cases have a higher level of Smoking, biochemical parameters, and DTAC score. Whereas, patients with NAFLD had a lower HDL and physical activity then the control groups. The highest tertiles of DTAC showed lower risk of NAFLD compared to the lowest tertiles. Also, this association was significant after controlling for potential confounders.

\section{Conclusions}

Our findings showed that a high DTAC was associated with a reduced risk of NAFLD in adult, suggesting that promoting a naturally elevated antioxidant capacity might help prevent the development of NAFLD. Further prospective studies should be conducted in this regard.

\section{Introduction}

Non-alcoholic fatty liver disease (NAFLD) is one of the major global health challenges that includes many of liver abnormalities such as hepatic steatosis, cirrhosis or hepatocellular carcinoma(1). This disease is characterized by accumulation of more than $5 \%$ of fat in the liver tissue cells in the absence of other risk factors; virus, immune or metabolic disorders and drug abuse(2). Worldwide estimated prevalence of NAFLD in the general population is $25 \%(3)$. In Iran, prevalence of NAFLD among children and adults has been reported $7 \%$ and $35 \%$, respectively(4).

Studies suggest that systemic inflammation or excessive levels of free radicals and also disrupting the antinvidant halanno in tha hadv in nthor wands oxidative stress, through increased lipid peroxidation in Loading [MathJax]/jax/output/CommonHTML/jax.js 
cell membranes may play an important key role in the pathogenesis of $\operatorname{NAFLD}(5,6)$. Thus, when antioxidant and anti-inflammatory defenses are becoming exhausted, a chronic state of liver diseases arises.

Various aspects of the effect of natural antioxidants found different in foods on the modulation of oxidative stress and disorders in the antioxidant system have been studied. $(7,8)$ Recently, in order to evaluate the total antioxidant capacity of foods and their beneficial health effects, the concept of dietary total antioxidant capacity (DTAC) was suggested as an indicator of cumulative power of antioxidants in the whole diet. Total antioxidant capacity diet is a strong correlation with serum total antioxidant capacity (9) and closely related to diet quality for determining the health outcomes in population(10).

Several evidences have suggested the potential link between DTAC and reduce the risk of chronic diseases such as diabetes(11), metabolic and oxidative stress markers(12), ulcerative colitis(13), blood pressure(14), and cardiovascular disease(15), which share common metabolic parameters with NAFLD. However, to our knowledge, the association between DTAC and risk of development NAFLD have not yet been investigated. Only recent a cross-sectional study in the steatohepatitis patients has declared that high DTAC score was related to low hepatic injury through reducing free radical production and, consequently, oxidative stress(16).

Considering the lack of convincing evidence regarding the association of DTAC with liver function, in this study we aimed to investigate the association between DTAC and risk of NAFLD in Iranian population in order to improve and reduce the burden of diseases.

\section{Subjects And Methods Participants}

This case-control study was performed on 158 patients with NAFLD and 357 healthy subjects who had been referred to the Hazrat Rasoul Hospital, Tehran, Iran during the recent years. NAFLD diagnosis was confirmed by chronic elevation of liver enzymes, absence of alcohol consumption, an ultrasonography scan of the liver compatible with NAFLD (Grade II, III) and exclusion of other etiologies of liver diseases. Case group patients were new cases and they were not treated before the study. Healthy individuals based on laboratory tests and the liver sonography (not suffering from any stages of hepatic steatosis) were considered as control group. Case and control were matched regarding age and gender. After entering into the study, information about demographic variables and enzymes level were obtained by completing the General Information Questionnaire. Also, the liver enzymes level checked after they came to the hospital, again. Then, for completing data on dietary intake and other information we called the patients to invite them to the research center in a special date. Participants with a history of certain diseases (diabetes, cardiovascular disease, myocardial infarction or stroke and cancer, viral hepatitis, Wilson's disease and autoimmune disorders of the liver) were excluded. Also, pregnant and lactating wnmen and cubierts with an arhitrarv cnerial diet were excluded. In the present study, the nutritionists Loading [MathJax]/jax/output/CommonHTML/jax.js 
were used as interviewer. So, all the patients responded completely to the survey questions. Also, in order to assess physical activity levels of participants, we used the General Practice Physical Activity Questionnaire (GPPAQ), a simple questionnaire reflecting an individual's current physical activity(17). This study was approved by the research council and ethics committee Iran University of Medical Sciences, Tehran, Iran.

\section{Anthropometric assessment}

Anthropometric measurements were conducted by a trained dietician. Weight was measured using a standard digital Seca scale (made in Germany), while participants wore minimum clothes and without shoes and recorded to the nearest $100 \mathrm{~g}$. Height was measured using a mounted tape in a standing relaxed shoulder position with no shoes to the nearest $0.5 \mathrm{~cm}$. Body mass index (BMI) was calculated as weight $(\mathrm{kg})$ divided by height in square meters $\left(\mathrm{m}^{2}\right)$.

\section{Measurement of biomarkers}

After 12 hours of fasting, $10 \mathrm{ml}$ of fasting blood was taken between 7 and 10 am of all participants. Blood samples were centrifuged for $10 \mathrm{~min}$ at $500 \mathrm{~g}$ and at $4{ }^{\circ} \mathrm{C}$ within $30-45 \mathrm{~min}$ of collection. The serums were shed in clean microbeads and stored at $-80^{\circ} \mathrm{C}$ until testing. Then, triglyceride, cholesterol, HDL-C, LDL-C, and glucose and cholesterol concentrations were measured by enzymatic methods using commercial kits (Pars Azmoon, Tehran, Iran). Alanine aminotransferase (ALT), aspartate aminotransferase (AST) were determined by commercially available enzymatic reagents (Pars Azmoon, Tehran, Iran) on auto analysis (BT-3000).

\section{Dietary assessment and DTAC calculation}

Trained dietitians administered the usual food intake during interview. A validated semiquantitative food frequency questionnaire (FFQ) that included 168 food items was used to assess the dietary intake(18). The consumption food items was calculated on a daily, weekly, or monthly. Then, data were transformed into the average monthly intake that finally were analyzed using the Nutritionist 4 software. The USDA portion sizes and household measures were used for each food. DTAC was calculated based on the ferric reducing-antioxidant power (FRAP) values that reported by US Department of Agriculture. The FRAP assay measures the ability of dietary antioxidants to reduce ferric to ferrous ions and is reported as mmol per 100 grams of foods(19).

\section{Statistical analysis}

Statistical analysis was conducted using Statistical Package Software for Social Science, version 21 (SPSS Inc., Chicago, IL, USA). The Kolmogorov-Smirnov's test and histogram chart was used for testing the normality of the data. Baseline characteristics and dietary intakes were expressed as mean \pm SD or median (25-75 interquartile range) for quantitative variables, and number and percentages for qualitative variables. Comparison of the data between two groups was done using independent sample t-test and chi square for continuous and categorical variables, respectively. 
Binary logistic regression was used to estimate odds ratios (ORs) and 95\% confidence intervals (Cls) adjusted for multiple covariates in different model. The data were presented as mean \pm standard deviation and odds ratio with $95 \%$ confidence interval, and in all results, the significance level was determined as $\mathrm{P}<0.05$.

\section{Result}

The mean \pm SD for age and BMI of the study participants were $43.9 \pm 5.9$ years (female percent $=58.7 \%$ ) and $30.5 \pm 2.6 \mathrm{Kg} . \mathrm{m} 2$. Table 1 demonstrates anthropometric and biochemical indices among case (non alcoholic fatty liver patients) and control groups. There were no significant differences between cases and controls in age; however, NAFLD patients had higher BMI and female's percentage compared with controls. Also, compared with the control, the NAFLD cases have a higher level of Smoking, FBS, triglyceride, LDL, total cholesterol, ALT, and DTAC score. Whereas, patients with NAFLD had a lower HDL and physical activity then the control groups. Dietary intake among the NAFLD Patients and Control Groups presented in Table 2. There was a significant difference in the intake of more micro and macronutrients between the two groups. Additionally, No significant differences were found between the cases and control in the intake of protein, fiber, selenium, and folate. The participants' characteristics and biochemical parameters across the tertiles of DTAC are presented in Table 3. The mean age was significantly increased across tertiles of DTAC $(p<0.002)$. While, weight, FBS, TG, and ALT were significantly decreased across tertiles of DTAC ( $p$ trend $<0.05)$. There were no significant differences in all other characteristics biochemical parameters across tertiles of DTAC.

\section{Table 1.}

Anthropometric

and

Biochemical

Parameters

Among the

Case (Non-

Alcoholic Fatty

Liver Patients)

and Control

Groups 


\begin{tabular}{|c|c|c|c|}
\hline \multirow[t]{2}{*}{ variables } & \multicolumn{2}{|c|}{ Groups, mean \pm SD } & \multirow[t]{2}{*}{$P$ value } \\
\hline & Case $(n=158)$ & Control $(n=357)$ & \\
\hline Women, \%(No) & $58.7(98)$ & $41.3(88)$ & $0.04^{\mathrm{a}}$ \\
\hline Age, y & $42.75 \pm 5.9$ & $45.13 \pm 5.9$ & $0.06^{b}$ \\
\hline Weight, kg & $94.44 \pm 15.6$ & $74.14 \pm 12.9$ & $<0.001^{b}$ \\
\hline $\mathrm{BMl}, \mathrm{kg} / \mathrm{m}^{2}$ & $33.19 \pm 3.1$ & $27.95 \pm 2.1$ & $<0.001^{b}$ \\
\hline Physical Activity (Met.min/wk) & $984.5 \pm 1241.5$ & $1563.2 \pm 1216.7$ & $<0.001 \mathrm{~b}$ \\
\hline Smoking (yes), n (\%) & $16(7.1)$ & $12(2.7)$ & 0.006 \\
\hline FBS, mg/dl & $109.29 \pm 7.6$ & $92.21 \pm 6.7$ & $<0.001^{b}$ \\
\hline Total cholesterol, mg/dl & $184.79 \pm 42.1$ & $182.85 \pm 40.1$ & $<0.001^{b}$ \\
\hline Triglyceride, mg/dl & $180.40 \pm 14.3$ & $130.99 \pm 12.2$ & $0.001^{b}$ \\
\hline $\mathrm{HDL}, \mathrm{mg} / \mathrm{dl}$ & $41.26 \pm 15.1$ & $48.5 \pm 17.3$ & $0.017^{b}$ \\
\hline LDL, mg/dl & $121.17 \pm 23.4$ & $109.14 \pm 13.0$ & $<0.001^{b}$ \\
\hline $\mathrm{ALT}, \mathrm{mg} / \mathrm{dl}$ & $58.50 \pm 24.1$ & $20.53 \pm 13.01$ & $<0.001^{b}$ \\
\hline DTAC, mmol TE/100 g & $17563.4 \pm 8247.2$ & $12323.6 \pm 5398.5$ & $<0.001^{b}$ \\
\hline \multicolumn{4}{|c|}{${ }^{a} P$ values are resulted from chi square } \\
\hline \multicolumn{4}{|c|}{${ }^{b} \mathrm{P}$ values are resulted from student t-test } \\
\hline
\end{tabular}


Table 2.Dietary Intake Among the Non Alcoholic Fatty Liver Patients, and Control Groups

\begin{tabular}{|llll|}
\hline variables & \multicolumn{2}{l}{ Groups, mean \pm SD } & \multirow{2}{*}{ P value $^{\mathrm{a}}$} \\
\cline { 2 - 4 } & Case $(\mathrm{n}=158)$ & Control $(\mathrm{n}=357)$ & \\
\hline Energy, kcal & $2741.8 \pm 819.89$ & $2427.67 \pm 798.09$ & 0.04 \\
\hline Macronutrients & & & \\
\hline Protein, gr & $97.33 \pm 34.37$ & $104.47 \pm 39.61$ & 0.06 \\
\hline Fat, gr & $103.44 \pm 40.11$ & $86.27 \pm 32.58$ & $<0.001$ \\
\hline Saturated fatty acid & $32.13 \pm 6.35$ & $26.87 \pm 6.42$ & 0.006 \\
\hline Carbohydrate, gr & $408.06 \pm 148.95$ & $378.44 \pm 112.63$ & 0.03 \\
\hline Fiber, gr & $46.36 \pm 18.25$ & $47.58 \pm 19.76$ & 0.5 \\
\hline Fruits & $249.38 \pm 190.29$ & $368.03 \pm 192.15$ & $<0.001$ \\
\hline vegetables & $269.36 \pm 183.63$ & $339.31 \pm 185.25$ & $<0.001$ \\
\hline Whole grain & $121.54 \pm 80.71$ & $134.44 \pm 79.91$ & $<0.001$ \\
\hline Refined grain & $420.41 \pm 140.35$ & $368.87 \pm 141.65$ & $<0.001$ \\
\hline fructose & $25.95 \pm 12.19$ & $23.52 \pm 10.33$ & 0.03 \\
\hline Micronutrients & & & $<0.0017$ \\
\hline Calcium (mg/d) & $1311.17 \pm 441.93$ & $1125.66 \pm 381.61$ & $<0.001$ \\
\hline Selenium(mg/d) & $135.39 \pm 56.28$ & $133.34 \pm 52.41$ & 0.717 \\
\hline Vitamin E (mg/d) & $13.22 \pm 5.866$ & $18.98 \pm 7.146$ & $<0.001$ \\
\hline Folate (mcg/d) & $615.53 \pm 182.21$ & $642.29 \pm 194.52$ & 0.176 \\
\hline Vitamin B12 (mcg/d) & $6.37 \pm 4.47$ & $5.36 \pm 4.42$ & 0.029 \\
\hline Vitamin D (mcg/d) & $2.03 \pm 1.80$ & $1.56 \pm 1.27$ & 0.03 \\
\hline Vitamin C (mg/d) & $231.92 \pm 144.06$ & $175.72 \pm 88.09$ & $<0.001$ \\
\hline ap values are resulted from student t-test & & \\
\hline
\end{tabular}


Table 3

Characteristics and Biochemical Parameters across tertiles of dietary total antioxidant capacity among the study population

\begin{tabular}{|c|c|c|c|c|}
\hline & \multicolumn{4}{|c|}{ Tertiles of dietary total antioxidant capacity } \\
\hline & $\mathrm{T} 1$ & $\mathrm{~T} 2$ & T3 & $\mathrm{P}^{\mathrm{a}}$ \\
\hline Age(year) & $37.0 \pm 8.3$ & $38.2 \pm 8.5$ & $39.5 \pm 9.6$ & 0.002 \\
\hline Weight, kg & $160(61.5)$ & $114(51.1)$ & $84(43.8)$ & 0.001 \\
\hline $\mathrm{BMI}, \mathrm{kg} / \mathrm{m}^{2}$ & $26.8 \pm 4.1$ & $26.7 \pm 4.4$ & $26.9 \pm 4.4$ & 0.687 \\
\hline Physical Activity (Met.min/wk) & $1462 \pm 862$ & $1454 \pm 850$ & $1368 \pm 939$ & 0.261 \\
\hline Smoking (yes), n (\%) & $9(3.5)$ & $11(4.9)$ & $8(4.2)$ & 0.722 \\
\hline FBS, mg/dl & $102.73 \pm 43.92$ & $95.20 \pm 36.79$ & $94.81 \pm 33.58$ & 0.001 \\
\hline Total cholesterol, mg/dl & $186.34 \pm 41.68$ & $182.20 \pm 43.70$ & $183.27 \pm 46.52$ & 0.804 \\
\hline Triglyceride, mg/dl & $155.56 \pm 114.17$ & $144.46 \pm 72.26$ & $141.09 \pm 79.87$ & $<0.001$ \\
\hline $\mathrm{HDL}, \mathrm{mg} / \mathrm{dl}$ & $47.09 \pm 12.02$ & $44.34 \pm 12.00$ & $47.52 \pm 14.61$ & 0.198 \\
\hline LDL, mg/dl & $113.42 \pm 31.53$ & $111.98 \pm 35.75$ & $111.11 \pm 35.77$ & 0.261 \\
\hline ALT, mg/dl & $39.24 \pm 51.22$ & $30.49 \pm 25.78$ & $29.78 \pm 38.03$ & $<0.001$ \\
\hline
\end{tabular}

The ORs and $95 \%$ Cls for NAFLD in the terrtiles of DTAC are shown in Table 4. Compared with participants in the lowest tertile of DTAC, those in the highest terrtile had a significantly lower OR for NAFLD (crude model: OR, $0.37 ; 95 \% \mathrm{Cl}, 0.24-0.58$ ), which remained significant after further adjustment for BMl; physical activity; education; and dietary intake of fiber, fat, energy, coffee, and biochemical parameters (model 3: OR, 0.22; $95 \% \mathrm{Cl}, 0.11-0.43$ ). 
Table 4

the association between Tertiles of dietary total antioxidant capacity and risk of NAFLD among the participants of study.

Tertiles of dietary total antioxidant capacity

P for trend

T1

T2

T3

DTAC

Case/Total $39 / 105 \quad 58 / 125 \quad 61 / 127$

Crude $\quad 1.00$ (Ref) $\quad 0.76(0.52-1.12) \quad 0.37(0.24-0.58) \quad 0.20(0.10-0.40) \quad<001$

Model 1* $\quad 1.00$ (Ref) $0.74(0.42-1.28) \quad 0.20(0.10-0.40) \quad 0.001$

$\begin{array}{lllll}\text { Model 2† } & 1.00(\text { Ref) } & 0.93(0.48-1.77) & 0.29(0.14-0.61) & 0.001\end{array}$

Model $3^{\ddagger} \quad 1.00$ (Ref) $\quad 0.68(0.39-1.19) \quad 0.22(0.11-0.43) \quad<0.001$

${ }^{*}$ Model 1: adjusted for age and sex

${ }^{\dagger}$ Model 2: adjusted for model 1 and BMI, physical activity, education, dietary intake of fiber, fat, energy, and coffee.

‡ Model 3: additionally adjusted for fasting blood sugar, TG, cholesterol, LDL-C, and HDL-C at baseline.

\section{Discussion}

The study investigated the association between DTAC and NAFLD in a case-control study. We found that DTAC had an inverse association with weight, FBS, TG, and ALT. Participants with higher DTAC score had a significantly lower OR for NAFLD compared with low DTAC score, after adjustment for BMl; physical activity; education level; and dietary intake of fiber, fat, energy, coffee, and biochemical parameters. In the present study, those with case had a lower DTAC score compared with the control group, suggesting that DTAC may be a potential predictor for the risk for development of NAFLD.

To the best of our knowledge, this is the first time that an inverse association between the DTAC and the risk of NAFLD has been documented. In the previous evidences, dietary TAC has been inversely associated with several chronic diseases such as cardiovascular diseases(15), cancer (20)diabetes (11), and metabolic disorders(12), which share common metabolic parameters with NAFLD. Also, in a casecontrol study that examined blood redox status with severity of non-alcoholic fatty liver disease(21), no significant correlation was found between diet antioxidant capacity with severity of non-alcoholic liver disease. Interestingly, there was no difference of diet antioxidant capacity score between the case and control groups in the mentioned report, and perhaps the results were due to this. However, thiobarbituric acid reactive substances was significantly associated with the likelihood of nonalcoholic fatty liver disease. independentlv diet's total antioxidant. Loading [MathJax]/jax/output/CommonHTML/jax.js 
Overall, an inverse associations between intake of antioxidant micronutrients and antioxidant-rich foods with risk of NAFLD was registered. For example, The DASH diet (Dietary Approaches to Stop Hypertension), dietary patterns with antioxidant-rich component based on fruit and vegetables, low-fat dairy products, and whole grains, demonstrated an inverse relationship with risk of $\operatorname{NAFLD}(22,23)$, as well as an inverse association in chronic diseases associated with NAFLD such as Cardiovascular disease and diabetes(24). Furthermore, coffee has been investigated as an antioxidant-rich nutrient as a risk reduction for NAFLD. So that, recently a meta-analysis suggested that coffee intake in a dosedependent manner reduces the risk of developing NAFLD(25). Also, the ability of other antioxidant-rich nutrient to increase plasma TAC and finally reduced risk of NAFLD through modified of oxidative stress, including bayberry juice(26), chocolate (27) onions(28), lettuce(29), or tomato products (30)was well revealed. The findings were confirmed even in complementary interventions with antioxidants. Besides, the beneficial effects of the combination of antioxidant compounds were also reported(31), as demonstrated the synergistic effect of Simultaneous administration and combination of one or more antioxidant supplements reducing risk of NAFLD(31).

Oxidative stress has been shown to be involved in the pathogenesis and development of lipid metabolism disorders and insulin resistance and can lead to NAFLD(32). Decreased antioxidant defense mechanisms can increase lipid peroxidation, damage cellular organs and enzymes, and cause insulin resistance(33). Thus, high dietary antioxidants improve lipid and glucose metabolism disorders and reduce risk of NAFLD by protecting liver cell $(8,16,33)$. Additionally, high intake of antioxidant-rich nutrient from plant foods, as part of a healthy diet, may confer health benefits not only through protection from oxidative damages(34), but also by providing fiber and antioxidant nutrient such as vitamin $D, C, E$, magnesium, which have advantageous on body mass index, and levels of serum lipids and also glucose(35). Also, the beneficial synergetic effects of antioxidants, fiber, potassium, magnesium, and other phytochemicals on prevention of NAFLD were observed in various researches $(35,36)$. On other hand, overweight and abdominal obesity have been suggested as one of the potential risk factors for the onset and progression of NAFLD(37). Dietary antioxidants provide a protective mechanism against obesity-related disorders, including inhibition of fat absorption, promotion of catabolism in the adipose tissue, inhibition of proliferation, differentiation, angiogenesis in preadipocytes, and induction of apoptosis in mature adipocytes (38).In line with these mechanisms te mean weight of patients were significantly decreased across tertiles of DTAC in our study.

The present study had some limitations that might affect the interpretation of the results. First, hospitalbased case-control studies. Second, using the 168-item FFQ questionnaire, cause tired participants' and bias in responded, which was resolved by a trained questioner. Third, some conditions of foods such as the growing condition, cultivation method, storage, processing and cooking condition, and assay method might affect the antioxidants content of the foods(19). Despite these limitations, this is the first study to examine the relationship between DTAC and NAFLD in a case control design and also and use of a validated diet history questionnaire to evaluate diet and dietary TAC. Furthermore, we controlled in the statistical analysis for several potential confounding variables. 


\section{Conclusion}

Our findings showed that a high DTAC was associated with a reduced risk of NAFLD in adult, suggesting that promoting a naturally elevated antioxidant capacity might help prevent the development of NAFLD. Encouragement of the consumption of a diet with high antioxidant capacity is important in nutritional interventions aimed at preventing NAFLD. However, Additional studies are needed to further investigate the association of DTAC intake with risk of NAFLD over time in other settings.

\section{Declarations}

\section{Acknowledgements}

We express our appreciation to the participants of this study.

\section{Disclosure statement}

None of the authors had any personal or financial conflicts of interest.

\section{Authors' contributions}

S.F, and Mh.S contributed in conception, design, and statistical analysis. Mh.S, contributed in data collection and manuscript drafting. F.Sh supervised the study.All authors approved the final version of the manuscript.

\section{Consent for publication}

All authors of this manuscript declared their consent for publication.

\section{Competing interests}

The authors declare that they have no competing interests.

\section{Funding}

No funding

\section{Availability of data and materials}


Raw data were generated by Iran University of medical sciences. Deriveddata supporting the findings of this study are available from the correspondingauthor upon request.

\section{Ethics approval and consent to participate}

The study received ethical approval from the Iran University of medical sciences and all participants in the study signed aninformed consent.

\section{Author information}

1Department of Nutrition, School of Public Health, Iran University of Medical Sciences, Tehran, Iran. 2Student Research Committee, Faculty of public health Branch, Iran University of medical sciences, Tehran, Iran

\section{References}

1. Petäjä EM, Yki-Järvinen H. Definitions of normal liver fat and the association of insulin sensitivity with acquired and genetic NAFLD-a systematic review. Int J Mol Sci. 2016;17(5):633.

2. Than NN, Newsome PN. A concise review of non-alcoholic fatty liver disease. Atherosclerosis. 2015;239(1):192-202.

3. Anderson EL, Howe LD, Jones HE, Higgins JP, Lawlor DA, Fraser A. The prevalence of non-alcoholic fatty liver disease in children and adolescents: a systematic review and meta-analysis. PloS one. 2015;10(10).

4. Naghavi M. Etiology of death in 18 provinces of Iran in year 2001. 21. Tehran: Ministry of Health and Medical Education IR Iran; 2003.

5. Ashraf N, Sheikh T. Endoplasmic reticulum stress and oxidative stress in the pathogenesis of nonalcoholic fatty liver disease. Free Radic Res. 2015;49(12):1405-18.

6. Cichoż-Lach H, Michalak A. Oxidative stress as a crucial factor in liver diseases. World journal of gastroenterology: WJG. 2014;20(25):8082.

7. Serafini M, Del Rio D. Understanding the association between dietary antioxidants, redox status and disease: is the total antioxidant capacity the right tool? Redox report. 2004;9(3):145-52.

8. Meydani M. Dietary antioxidants modulation of aging and immune-endothelial cell interaction. Mech Ageing Dev. 1999;111(2-3):123-32.

9. Cao G, Prior RL. Comparison of different analytical methods for assessing total antioxidant capacity of human serum. Clinical chemistry. 1998;44(6):1309-15.

10. Wu X, Gu L, Holden J, Haytowitz DB, Gebhardt SE, Beecher G, et al. Development of a database for total antioxidant capacity in foods: a preliminary study. Journal of Food composition analysis. 
11. Mancini FR, Affret A, Dow C, Balkau B, Bonnet F, Boutron-Ruault M-C, et al. Dietary antioxidant capacity and risk of type 2 diabetes in the large prospective E3N-EPIC cohort. Diabetologia. 2018;61(2):308-16.

12. Hermsdorff HHM, Puchau B, Volp ACP, Barbosa KB, Bressan J, Zulet M, et al. Dietary total antioxidant capacity is inversely related to central adiposity as well as to metabolic and oxidative stress markers in healthy young adults. Nutrition metabolism. 2011;8(1):59.

13. Koch TR, Yuan L-X, Stryker SJ, Ratliff P, Telford GL, Opara EC. Total antioxidant capacity of colon in patients with chronic ulcerative colitis. Digestive diseases sciences. 2000;45(9):1814-9.

14. Villaverde P, Lajous M, MacDonald C-J, Fagherazzi G, Bonnet F, Boutron-Ruault M-C. High dietary total antioxidant capacity is associated with a reduced risk of hypertension in French women. Nutrition journal. 2019;18(1):31.

15. Rautiainen S, Levitan EB, Orsini N, Åkesson A, Morgenstern R, Mittleman MA, et al. Total antioxidant capacity from diet and risk of myocardial infarction: a prospective cohort of women. Am J Med. 2012;125(10):974-80.

16. de Oliveira DG, de Faria Ghetti F, Moreira APB, Hermsdorff HHM, de Oliveira JM, de Castro LEVV. Association between dietary total antioxidant capacity and hepatocellular ballooning in nonalcoholic steatohepatitis: a cross-sectional study. Eur J Nutr. 2019;58(6):2263-70.

17. Ahmad S, Harris T, Limb E, Kerry S, Victor C, Ekelund U, et al. Evaluation of reliability and validity of the General Practice Physical Activity Questionnaire (GPPAQ) in 60-74 year old primary care patients. 2015;16(1):113.

18. Azadbakht L, Esmaillzadeh A. Red meat intake is associated with metabolic syndrome and the plasma C-reactive protein concentration in women. J Nutr. 2009;139(2):335-9.

19. Haytowitz DB, Bhagwat S. USDA database for the oxygen radical absorbance capacity (ORAC) of selected foods, Release 2. US Department of Agriculture. 2010:10-48.

20. Vece MM, Agnoli C, Grioni S, Sieri S, Pala V, Pellegrini N, et al. Dietary total antioxidant capacity and colorectal cancer in the Italian EPIC cohort. PLoS One. 2015;10(11).

21. Georgoulis M, Fragopoulou E, Kontogianni MD, Margariti A, Boulamatsi O, Detopoulou P, et al. Blood redox status is associated with the likelihood of nonalcoholic fatty liver disease irrespectively of diet's total antioxidant capacity. Nutr Res. 2015;35(1):41-8.

22. Hekmatdoost A, Shamsipour A, Meibodi M, Gheibizadeh N, Eslamparast T, Poustchi H. Adherence to the dietary approaches to stop hypertension (DASH) and risk of nonalcoholic fatty liver disease. Int J Food Sci Nutr. 2016;67(8):1024-9.

23. Xiao M-L, Lin J-S, Li Y-H, Liu M, Deng Y-Y, Wang C-Y, et al. Adherence to the Dietary Approaches to Stop Hypertension (DASH) diet is associated with lower presence of non-alcoholic fatty liver disease in middle-aged and elderly adults. Public Health Nutrition. 2019:1-9.

24. Liese AD, Nichols M, Sun X, D'Agostino RB, Haffner SM. Adherence to the DASH Diet is inversely associated with incidence of type 2 diabetes: the insulin resistance atherosclerosis study. Diabetes 
25. Chen Y-P, Lu F-B, Hu Y-B, Xu L-M, Zheng M-H, Hu E-D. A systematic review and a dose-response meta-analysis of coffee dose and nonalcoholic fatty liver disease. Clin Nutr. 2019;38(6):2552-7.

26. Guo H, Zhong R, Liu Y, Jiang X, Tang X, Li Z, et al. Effects of bayberry juice on inflammatory and apoptotic markers in young adults with features of non-alcoholic fatty liver disease. Nutrition. 2014;30(2):198-203.

27. Malhi H, Loomba R. dark chocolate may improve NAFLD and metabolic syndrome by reducing oxidative stress. Aliment Pharmacol Ther. 2016;44(5):533.

28. Emamat H, Foroughi F, Eini-Zinab H, Taghizadeh M, Rismanchi M, Hekmatdoost A. The effects of onion consumption on treatment of metabolic, histologic, and inflammatory features of nonalcoholic fatty liver disease. Journal of Diabetes Metabolic Disorders. 2015;15(1):25.

29. Nikkhah-Bodaghi M, Ghanavati M, Hekmatdoost A. Polyphenol intakes and risk of impaired lipid profile, elevated hepatic enzymes and nonalcoholic fatty liver disease. Nutrition \& Food Science. 2019.

30. García-Alonso F, González-Barrio R, Martín-Pozuelo G, Hidalgo N, Navarro-González I, Masuero D, et al. A study of the prebiotic-like effects of tomato juice consumption in rats with diet-induced nonalcoholic fatty liver disease (NAFLD). Food function. 2017;8(10):3542-52.

31. Sawangjit R, Chongmelaxme B, Phisalprapa P, Saokaew S, Thakkinstian A, Kowdley KV, et al. Comparative efficacy of interventions on nonalcoholic fatty liver disease (NAFLD): A PRISMAcompliant systematic review and network meta-analysis. Medicine. 2016;95(32).

32. Narasimhan S, Gokulakrishnan K, Sampathkumar R, Farooq S, Ravikumar R, Mohan V, et al. Oxidative stress is independently associated with non-alcoholic fatty liver disease (NAFLD) in subjects with and without type 2 diabetes. Clinical biochemistry. 2010;43(10-11):815-21.

33. Liu W, Baker SS, Baker D, Zhu R. L. Antioxidant mechanisms in nonalcoholic fatty liver disease. Curr Drug Targets. 2015;16(12):1301-14.

34. Valko M, Leibfritz D, Moncol J, Cronin MT, Mazur M, Telser J. Free radicals and antioxidants in normal physiological functions and human disease. Int J Biochem Cell Biol. 2007;39(1):44-84.

35. Mouzaki M, Allard JP. The role of nutrients in the development, progression, and treatment of nonalcoholic fatty liver disease. Journal of clinical gastroenterology. 2012;46(6):457-67.

36. Dreher ML. Dietary Patterns, Foods, Nutrients and Phytochemicals in Non-Alcoholic Fatty Liver Disease. Dietary Patterns and Whole Plant Foods in Aging and Disease: Springer; 2018. p. $291-311$.

37. Jakobsen MU, Berentzen T, Sørensen T, Overvad K. Abdominal obesity and fatty liver. Epidemiol Rev. 2007;29(1):77-87.

38. Barnard ND, Scialli AR, Turner-McGrievy G, Lanou AJ, Glass J. The effects of a low-fat, plant-based dietary intervention on body weight, metabolism, and insulin sensitivity. Am J Med. 2005;118(9):991-7. 